Supporting Information

\title{
Smart Glove Integrated with Tunable MWNTs/PDMS Fibers Made of a One-Step Extrusion Method for Finger Dexterity, Gesture, and Temperature Recognition
}

Yingchun Li, ${ }^{\dagger \ddagger}$ Chunran Zheng, ${ }^{\ddagger}, \S$ Shuai Liu, ${ }^{\dagger,}$ Liang Huang, ${ }^{\dagger, \ddagger}$ Tianshu Fang, ${ }^{\dagger \ddagger}$ Jasmine Xinze $\mathrm{Li}^{\ddagger}{ }^{\ddagger}$ Feng $\mathrm{Xu},{ }^{\dagger,}$ Fei $\mathrm{Li}^{*},+, \ddagger$

'The Key Laboratory of Biomedical Information Engineering of Ministry of Education, School of Life Science and Technology, Xi'an Jiaotong University, Xi'an 710049, P. R. China

${ }^{*}$ Bioinspired Engineering and Biomechanics Center (BEBC), Xi'an Jiaotong University, Xi'an 710049 , P. R. China

${ }^{\S}$ The School of Electronic and Information Engineering, Xi'an Jiaotong University, Xi'an 710049, P. R. China

*E-mail: feili@mail.xjtu.edu.cn 


\section{S1. Schematic of the smart glove}

The smart glove was integrated by a fabric glove with sensors for data collection and a custom-made microprocessor chip for decoding and transmitting the information of finger dexterity, gesture language and temperature. As shown in Figure S1, the layout of the microprocessor chip was based on a minimum system board (ATMEGA2560), including microprocessor and output modules. Data acquisition circuit, calibration circuit and filter circuit play critical roles in the microprocessor module. The MWNTs/PDMS fibers acted as variable resistances along with temperature and strain were linked to the data acquisition circuit to obtain digital signals through Analog Digital Converter (ADC) control. A calibration circuit was introduced using an extra resistance to reduce errors from power loss in data acquisition circuit. In addition, a filter circuit was added to avoid signal distortion and improve accuracy. After that, the correction digital signals transferred to output modules and output through various forms, such as screen display, phonetic system (e.g., loudspeaker and earphone) and bluetooth transmission.

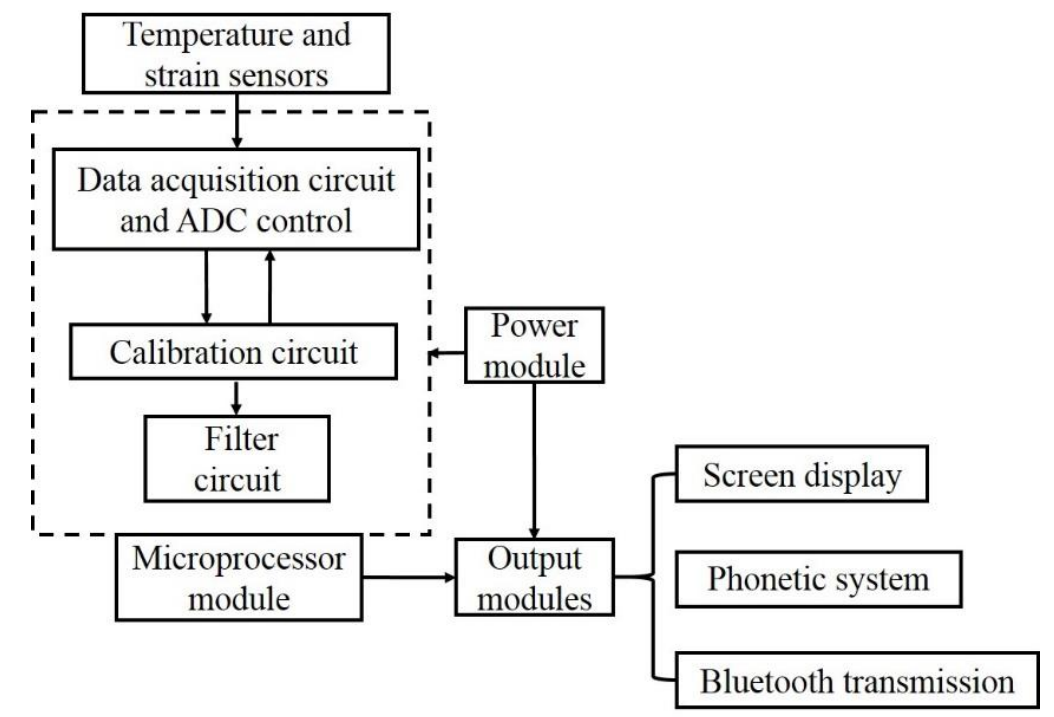

Figure S1. Schematic of the fabricated smart glove.

\section{S2. Circuit diagram of the hardware design}

The smart glove was integrated with nine strain sensors installed on the reverse side of five fingers, a temperature sensor on the front of index finger, and a trilaminar 
microprocessor chip on the back of hand. The circuit diagram of the exploded views of the trilaminar microprocessor chip is shown in Figures S2-S3. The data acquisition circuit was connected by a thin and flexible varnished wire, in which the sensors made of MWNTs/PDMS fibers acted as variable resistances along with temperature and strain and were linked to the flexible wire through tying a knot. Other ten voltage dividers were installed in series with the sensors to convert their dynamically-varying resistance into measuring voltages and obtain digital signals through ADC control (Figure S3a). An extra resistance was designed to parallel connect with the ten sensors as a calibration circuit for reducing errors from power loss in data acquisition circuit on a minimum system board based on the open-source ATMEGA2560 (Figure S2). A filter circuit was also added to avoid signal distortion and improve accuracy (Figure S3b). A layout of the connection type between the key units and power modules is shown in Figure S3c (for microcontroller, screen and phonetic system) and Figure S3d (for Bluetooth system). Figure S3e displays a program interface connecting a reset unit for providing the customized algorithms to the chip. Due to the different voltage requirements of various modules, a direct voltage conversion model transforming the voltage of $5 \mathrm{~V}$ to $3.3 \mathrm{~V}$ (Figure S3f) and a filter circuit (Figure S3g) were designed for compatible and stable power supply. 


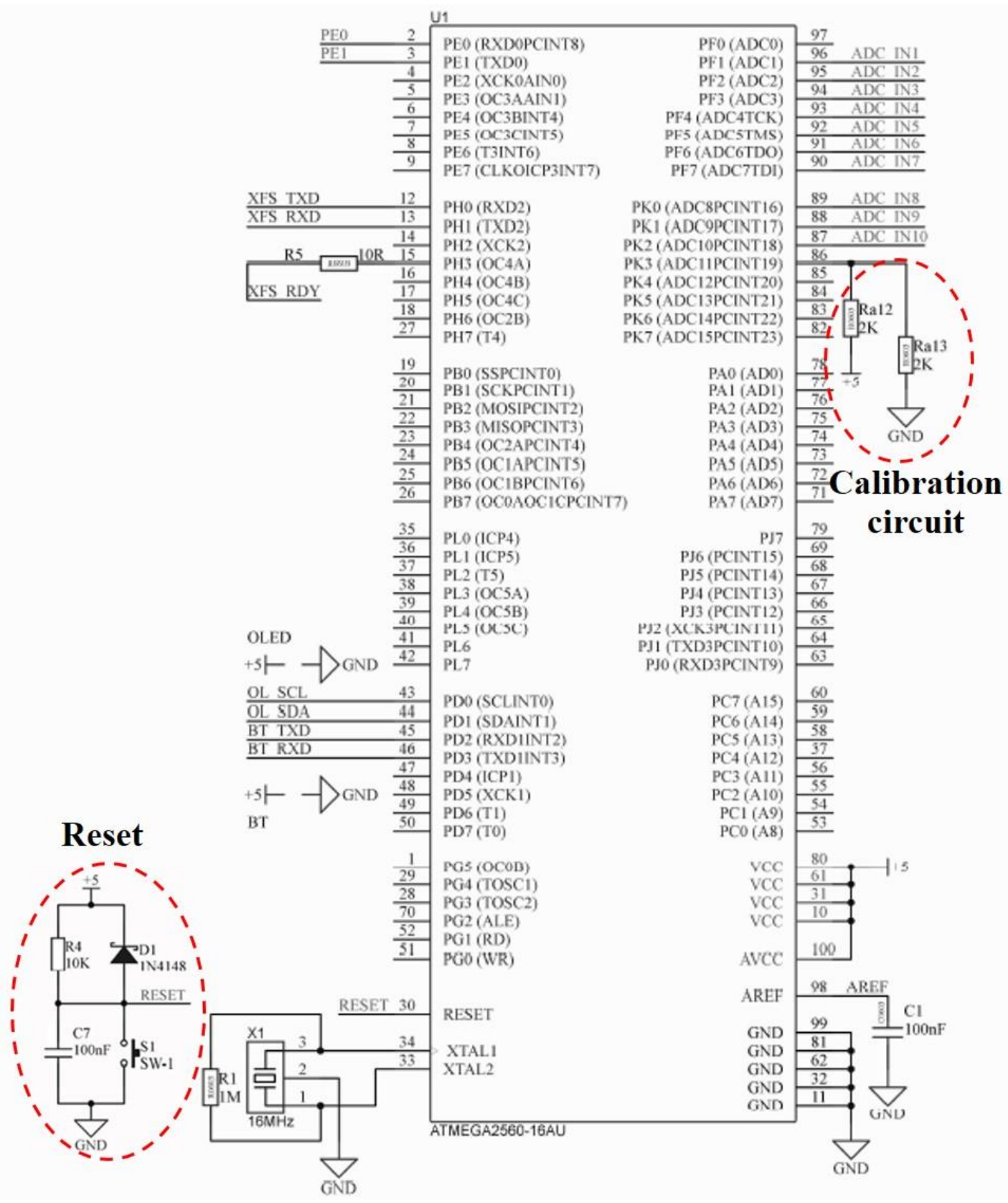

Figure S2. The circuit diagram of the minimum system board based on the open-source ATMEGA2560. 
a

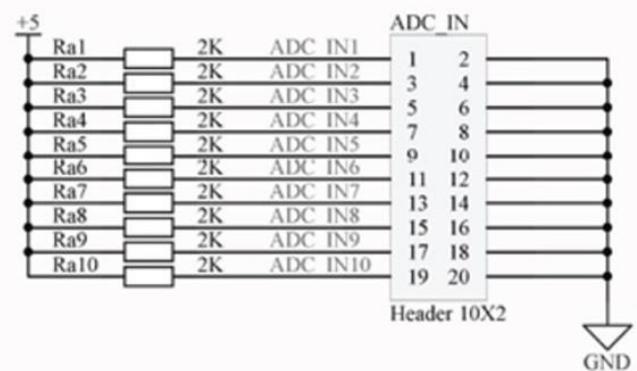

c

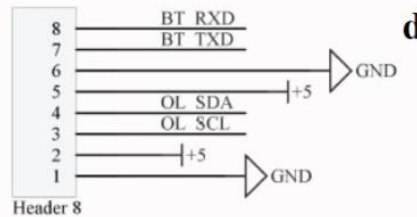

b
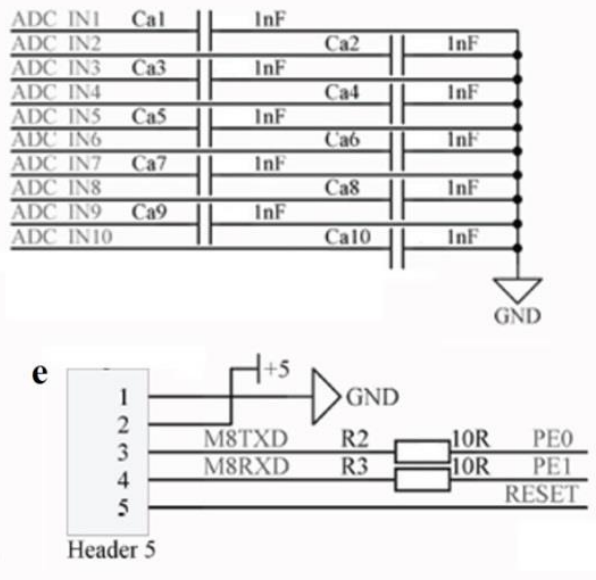

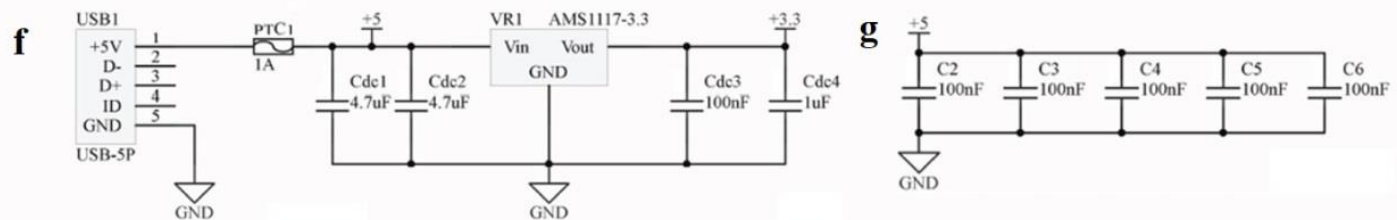

Figure S3. The component details of the minimum system board. (a) Data acquisition circuit; (b) Filter circuit for the data acquisition circuit; (c) The connection type between the units of microcontroller, screen, phonetic system and power modules; (d) The connection type between bluetooth and power modules; (e) Program interface; (f) a direct voltage conversion model; (g) a filter circuit of power module.
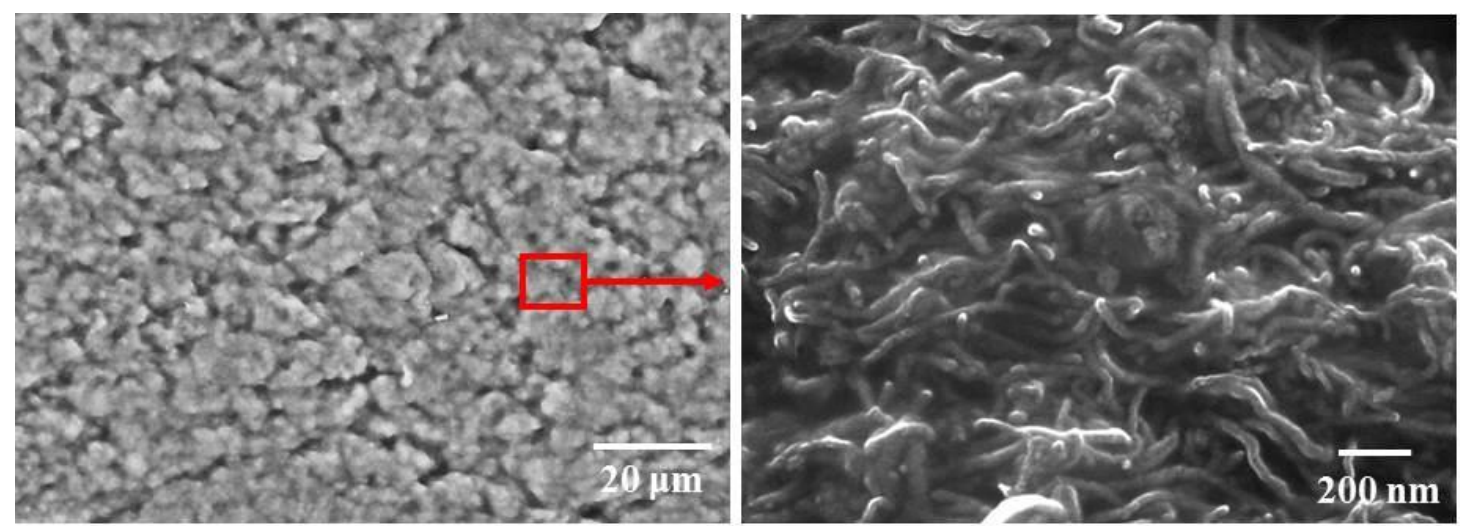

Figure S4. SEM images of MWNTs/PDMS fiber after 20000 cycles test with $50 \%$ tension. 


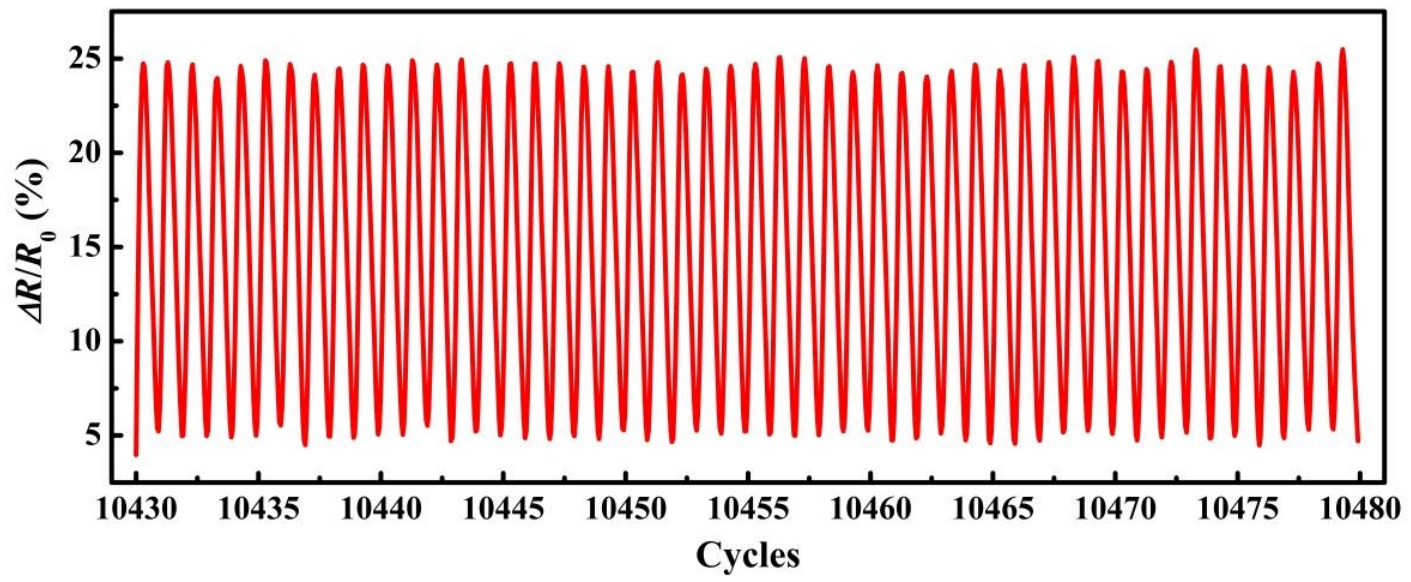

Figure S5. $\Delta R / R_{0}$ values of the MWNTs/PDMS fiber extracted from the cyclic curves of 20,000 cycles. 